\title{
What is the Impact of Sex on Cardiovascular Disease Risk Factors in Patients with Chronic Kidney Diseases in China: A Cross-sectional Study
}

\author{
Zu-feng Wang \\ Huazhong University of Science and Technology Tongji Medical College \\ Nan-hui Zhang \\ Huazhong University of Science and Technology Tongji Medical College \\ Ran Luo \\ Tongji Hospital of Tongji Medical College of Huazhong University of Science and Technology \\ Yi-chun Cheng \\ Tongji Hospital of Tongji Medical College of Huazhong University of Science and Technology \\ Kang-lin Guo \\ Huazhong University of Science and Technology Tongji Medical College \\ Shuwang Ge ( $\nabla$ geshuwang@tjh.tjmu.edu.cn ) \\ 0288 \\ Gang Xu \\ Tongji Hospital of Tongji Medical College of Huazhong University of Science and Technology
}

Tongji Hospital of Tongji Medical College of Huazhong University of Science and Technology https://orcid.org/0000-0002-5511-

\section{Research article}

Keywords: Cardiovascular risk factors, chronic kidney diseases, lipid profiles, sex, visceral adiposity

Posted Date: August 11th, 2020

DOI: https://doi.org/10.21203/rs.3.rs-41699/v1

License: (c) (i) This work is licensed under a Creative Commons Attribution 4.0 International License. Read Full License 


\section{Abstract}

Background: It is controversial about the sex differences in the association of chronic kidney diseases (CKD) and cardiovascular disease (CVD) risk. Thus, we examined CVD risk makers of CKD and non-CKD men and women in China, especially some "nontraditional" ones.

Methods: This cross-sectional study used 7999 participants from the China Health and Nutrition Survey in 2009. This study examined the "traditional" risk factors and of CVD, such as lipoprotein cholesterol (LDL-C), total cholesterol (TC) and non-high density lipoprotein cholesterol(non-HDL-C).Also, the "non-traditional" risk factors of CVD were calculated, such as lipoprotein (a) (Lp(a)), white blood cell (WBC) count, visceral adiposity index (VAI) and lipid accumulation product (LAP).

Results: Compared with men with CKD, higher levels of TC and LDL-C were observed in women with CKD. Furthermore, compared with men with CKD, the relative difference of WBC count was greater between women with CKD and their non-CKD ones. Meanwhile, the level of LAP and VAI of women with CKD were higher than men with CKD, which indicate the visceral obese. We also observed that the sex by CKD status interactions were statistically significant for TC, LDL-C, non-HDL-C, LAP, VAl and Lp(a) (all p <0.05). After adjusted the covariates, the sex differences effect on CVD risk factors among CKD patients couldn't be eliminated as well.

Conclusions: In CKD situation, women had greater lipid profiles and put on more visceral adiposity than men, which may indicate a higher CVD risk of women with CKD.

\section{Background}

In the previous studies, chronic kidney disease (CKD) has considered as an important public health problem and received lots of attention (1). In China, the prevalence of CKD in adults has reached 10.8\%(2). Besides, cardiovascular disease (CVD) has shown an increasing incidence and it is one of the most important causes of death in patients with CKD (3). At the same time, there many risk factors for CVD and CKD is one of them (4), and the risk of CVD increases with the development of CKD (5). CVD is interrelating with kidney disease closely, which is called cardiorenal syndrome, in which one organ's disease leads to the dysfunction of the other, ultimately causing both organ failed (6).

Recent studies demonstrated that advanced age, hypertension, diabetes, and dyslipidemia were considered as the CVD risk factors (7). Some lipid markers and inflammatory markers, including Lipoprotein (a)(Lp(a))(8) and white blood cell (WBC) count(9), were known as "non-traditional" risk factors of CVD(10). Besides, visceral adiposity index (VAl) and lipid accumulation product (LAP) were indicating the abdominal obesity and abdominal obesity was deeply related with CVD (11).Thus, VAl and LAP are considered as "non-traditional" risk factors of CVD as well(12). Due to the different living habits (13) and incidence of lipid metabolism disorder syndrome(14) between different genders, men were reported to have larger value of traditional risk factors (15), however, few researches focus on the "non-traditional" ones.

It is reported that $43.9 \%$ of CKD patients in China are men and $56.1 \%$ of them are women, indicating that there are more women patients with CKD in China(2). Population-based studies have showed that there some difference between genders of CKD epidemiology, in which CKD affected more women than men(16). Particularly, the differences between these markers mentioned above in men and women with CKD are short of research. We need to arouse the importance of the difference between different genders with CKD for CVD risk factors in order to give better treatment to CKD patients. Thus, we designed this study to explain the gender differences in CVD risk factors in CKD patients, including some "non-traditional" ones.

\section{Methods}

\section{Participants}

The data used in this study was from a database called China Health and Nutrition Survey (CHNS). This is a household-based and longitudinal survey in China. There were 8 survey rounds of CHNS and conducted in different years. Besides, the participants were from nine provinces (Heilongjiang, Liaoning, Henan, Jiangsu, Hubei, Shandong, Hunan, Guizhou and Guangxi), and more than half of China's population is included in this study, a great difference can be found about the health status, economic development and 
geography. The Lots of institutions supported this survey as mentioned in previous study(17) and all participant were provided a written informed consent (18).

Since CHNS began to collect the fasting blood in 2009, data from CHNS 2009 were used in this study. At the 2009exam, 10243 adult respondents were surveyed with 1120 did not give blood. Meanwhile, there 64 participants were pregnant and 805 participants' age was under 18, there 8254 participants with fasting blood samples finally. There also some different exclusion criteria including participants without the information of body mass index (BMI), age or waist circumference (WC) and with extreme triglycerides (TG) $(>500 \mathrm{mg} / \mathrm{dl}), \mathrm{BMI}\left(\geq 40 \mathrm{~kg} / \mathrm{m}^{2}\right.$ ) or high-density lipoprotein cholesterol (HDL-C) (>100 mg/dl) values. At the same time, in order to eliminate the different effects caused by the curing of CVD risk factors on the result of this study between different genders, participants were using anti-diabetic agent, lipid-lowering agent and anti-hypertension agent were excluded as well. This analysis included 7999 participants in the end.

\section{Measurements}

The stuff of CHNS used standard protocols from the World Health Organization (WHO) to measure participants' height, weight, systolic/diastolic BP and WC. Also, they used a calibrated beam scale to measure the participants' weight with a light clothing and portable SECA stadiometer to measure height. Using an inelastic tape to measure WC at middle between the top of the exhalation ilium and the bottom of chest. BMI was equal to the value using square of height (in meters) to divide weight (in kilograms). Meanwhile, trained technicians used mercury manometers to measure BP after a rest of ten minutes three times and averaging the three readings of $\mathrm{BP}$ in the end.

\section{Measurements of biochemical}

All tested samples were collected after a more than 8-hour overnight fast. We analyzed total samples with strict quality control in a lab of Beijing. Picric acid method was used to measure serum creatinine (Scr). GODPAP method was used to measure fasting plasm glucose (FPG). Hitachi 7600 automated analyzer was used to measure all lipids parameters, including, TG, low density lipoprotein cholesterol [LDL-C] and HDL-C. The value of non-HDL-C equaled to TC minus HDL-C. Measured Lp (a) with an immunoturbidimetric method. Also, apolipoprotein B (apo B), apolipoprotein a1 (apo a1), alanine aminotransferase (ALT), uric acid (UA), fasting insulin concentration and hypersensitive C-reactive protein (hs-CRP) were measured accurately with proper methods. Details on these procedures have been described previously(17). With drawing a conclusion of data from Chinese chronic kidney disease, estimated glomerular filtration rate (eGFR) was calculated with an equation developed by adaptation of the Modification of Diet in Renal Disease (MDRD) equation: eGFR $=175 \times \mathrm{Scr}^{-1.234} \times$ age $^{-0.179}$ [if female, $\times 0.79$ ] (19). CKD is defined as having eGFR< $60 \mathrm{ml} / \mathrm{min} / 1.73 \mathrm{~m}^{2}(20)$.

The LAP was calculated as LAP (18): Men: [WC (cm) - 65] × [TG (mmol/l)]; Women: [WC (cm) - 58] × [TG (mmol/l)]. And in order to avoid the nonpositive values of LAP, we eliminated men/women with WC values less than 65/58. We calculated homeostasis model assessment of insulin resistance (HOMA-IR) (21) with the formula: HOMA-IR = fasting insulin (micro-international units per milliliter) $\times$ FPG (millimoles per liter)/22.5. The triglycerides and glucose index (TyG) were calculated by the formula: TyG index(22): Ln [TG $(\mathrm{mg} / \mathrm{dl}) \times \mathrm{FPG}(\mathrm{mg} / \mathrm{dl}) / 2]$. Also, we used the formulas to calculated $\mathrm{VAl}(23)$, in which men were used the formula : [WC/39.68 + $(1.88 \times \mathrm{BMI})] \times(\mathrm{TG} / 1.03) \times(1.31 / \mathrm{HDL})$; and the formula for women was : $[\mathrm{WC} / 36.58+(1.89 \times \mathrm{BMI})] \times(\mathrm{TG} / 0.81) \times(1.52 / \mathrm{HDL})$.

\section{Statistical analysis}

We used SPSS software (version 25.0 for windows; SPSS, Chicago, IL, USA) to conducted the statistical analysis. And control persons were those participants who had eGFR $\geq 60 \mathrm{ml} / \mathrm{min} / 1.73 \mathrm{~m}^{2}$. All continuous variables were calculated as mean \pm standard deviation (SD). We used two-way analysis of covariance to computed CKD status and gender as the two main effects in five tables in this study. We also conducted the statistical analysis of the association of sex $\times$ CKD interactions with CVD risk factors. A logtransformed (natural logarithm) was used in insulin to approximate normal distributions before conducting analyses. A two-sided Pvalue $<0.05$ was regarded as statistically significant.

\section{Results}


Table 1

Non-adjusted mean levels of studied cardiovascular disease risk factors

\begin{tabular}{|c|c|c|c|c|c|c|c|c|c|}
\hline & Men & & & Women & & & $\begin{array}{l}\text { Men vs } \\
\text { Women }\end{array}$ & & \\
\hline & CKD & non-CKD & $P^{*}$ & CKD & non-CKD & $\mathbf{P}^{*}$ & $\mathrm{P}(\mathrm{CKD})$ & $\begin{array}{l}\text { P(non- } \\
\text { CKD) }\end{array}$ & $\mathrm{P}(\text { Interaction })^{\&}$ \\
\hline $\mathbf{N}$ & 290 & 3399 & & 327 & 3983 & & & & \\
\hline Age(years) & $66.8 \pm 0.7$ & $49.6 \pm 0.3$ & $\begin{array}{l}< \\
0.001\end{array}$ & $67.1 \pm 0.7$ & $49.4 \pm 0.2$ & $\begin{array}{l}< \\
0.001\end{array}$ & 0.764 & 0.500 & 0.764 \\
\hline $\mathrm{BMI}\left(\mathrm{kg} / \mathrm{m}^{2}\right)$ & $23.5 \pm 0.2$ & $23.2 \pm 0.1$ & 0.209 & $23.6 \pm 0.2$ & $23.3 \pm 0.1$ & 0.180 & 0.705 & 0.183 & 0.705 \\
\hline WC(cm) & $85.9 \pm 0.6$ & $84.1 \pm 0.2$ & 0.005 & $82.8 \pm 0.6$ & $81.1 \pm 0.2$ & 0.004 & $<0.001$ & $\begin{array}{l}< \\
0.001\end{array}$ & $<0.001$ \\
\hline SBP(mmHg) & $137.6 \pm 1.2$ & $125.0 \pm 0.3$ & $\begin{array}{l}<.001 \\
0.00\end{array}$ & $137.4 \pm 1.3$ & $122.5 \pm 0.3$ & $\begin{array}{l}<.001 \\
0.00\end{array}$ & 0.939 & $<0.001$ & 0.939 \\
\hline DBP(mmHg) & $84.7 \pm 0.7$ & $81.7 \pm 0.2$ & $\begin{array}{l}< \\
0.001\end{array}$ & $81.6 \pm 0.7$ & $78.8 \pm 0.2$ & $\begin{array}{l}< \\
0.001\end{array}$ & 0.002 & $\begin{array}{l}< \\
0.001\end{array}$ & 0.002 \\
\hline $\begin{array}{l}\text { eGFR(ml/min } \\
\left.\text { per1.73 } \mathrm{m}^{2}\right)\end{array}$ & $51.9 \pm 0.5$ & $84.0 \pm 0.3$ & $<0.001$ & $50.7 \pm 0.5$ & $84.4 \pm 0.2$ & $<0.001$ & 0.133 & 0.332 & 0.133 \\
\hline $\mathrm{TG}(\mathrm{mmol} / \mathrm{l})$ & $1.7 \pm 0.1$ & $1.6 \pm 0.0$ & 0.070 & $1.8 \pm 0.1$ & $1.4 \pm 0.0$ & $\begin{array}{l}< \\
0.001\end{array}$ & 0.290 & $\begin{array}{l}<.001 \\
0.01\end{array}$ & 0.135 \\
\hline $\mathrm{TC}(\mathrm{mmol} / \mathrm{l})$ & $4.9 \pm 0.1$ & $4.8 \pm 0.0$ & 0.001 & $5.4 \pm 0.1$ & $4.8 \pm 0.0$ & $\dot{0} 001$ & $<0.001$ & $\dot{0} 001$ & $<0.001$ \\
\hline $\mathrm{HDL}-\mathrm{C}(\mathrm{mmol} / \mathrm{l})$ & $1.4 \pm 0.0$ & $1.4 \pm 0.0$ & 0.762 & $1.5 \pm 0.0$ & $1.5 \pm 0.0$ & 0.945 & 0.001 & $<0.001$ & 0.001 \\
\hline LDL-C(mmol/l) & $3.1 \pm 0.1$ & $2.9 \pm 0.0$ & 0.003 & $3.5 \pm 0.1$ & $3.0 \pm 0.0$ & $\begin{array}{l}< \\
0.001\end{array}$ & $<0.001$ & 0.016 & $<0.001$ \\
\hline TG/HDL & $1.4 \pm 0.1$ & $1.3 \pm 0.0$ & 0.091 & $1.4 \pm 0.1$ & $1.1 \pm 0.0$ & $\begin{array}{l}<.001 \\
0.00\end{array}$ & 0.701 & $<0.001$ & 0.701 \\
\hline $\begin{array}{l}\text { non- } \\
\text { HDL(mmol/l) }\end{array}$ & $3.6 \pm 0.1$ & $3.4 \pm 0.0$ & 0.001 & $3.9 \pm 0.1$ & $3.4 \pm 0.0$ & $\dot{0} 001$ & $<0.001$ & 0.589 & $<0.001$ \\
\hline Apo B(g/l) & $1.0 \pm 0.0$ & $0.9 \pm 0.0$ & $\begin{array}{l}< \\
0.001\end{array}$ & $1.1 \pm 0.3$ & $0.9 \pm 0.3$ & $\begin{array}{l}< \\
0.001\end{array}$ & $<0.001$ & 0.467 & $<0.001$ \\
\hline ApoB/apo a1 & $0.9 \pm 0.0$ & $0.8 \pm 0.0$ & 0.048 & $0.9 \pm 0.4$ & $0.8 \pm 0.3$ & $\begin{array}{l}<.001 \\
0.00\end{array}$ & 0.235 & $\begin{array}{l}<.001 \\
0.00\end{array}$ & 0.235 \\
\hline FPG(mmol/l) & $5.9 \pm 0.1$ & $5.3 \pm 0.0$ & $\dot{0} 001$ & $6.0 \pm 0.1$ & $5.3 \pm 0.0$ & $\dot{0} 001$ & 0.534 & 0.001 & 0.443 \\
\hline $\begin{array}{l}\mathrm{HbA} 1 \mathrm{c} \\
(\mathrm{mmol} / \mathrm{mol})\end{array}$ & $5.8 \pm 0.1$ & $5.6 \pm 0.1$ & 0.001 & $5.9 \pm 0.1$ & $5.6 \pm 0.0$ & $\dot{0} 001$ & 0.164 & 0.109 & 0.164 \\
\hline HOMA-IR & $6.5 \pm 0.1$ & $3.6 \pm 0.1$ & $\begin{array}{l}<.001 \\
0.001\end{array}$ & $6.5 \pm 0.8$ & $3.4 \pm 0.1$ & $\begin{array}{l}<.001 \\
0.001\end{array}$ & 0.961 & 0.196 & 0.961 \\
\hline Insulin & $1.1 \pm 0.0$ & $1.0 \pm 0.0$ & $\begin{array}{l}<.001 \\
0.001\end{array}$ & $1.1 \pm 0.0$ & $1.0 \pm 0.0$ & $\begin{array}{l}<.001 \\
0.001\end{array}$ & 0.997 & 0.261 & 0.997 \\
\hline Tyg index & $8.8 \pm 0.0$ & $8.6 \pm 0.0$ & $\begin{array}{l}< \\
0.001\end{array}$ & $8.9 \pm 0.0$ & $8.5 \pm 0.0$ & $\begin{array}{l}< \\
0.001\end{array}$ & 0.159 & $\begin{array}{l}< \\
0.001\end{array}$ & 0.159 \\
\hline ALT & $22.7 \pm 1.0$ & $27.7 \pm 0.4$ & $\begin{array}{l}< \\
0.001\end{array}$ & $21.7 \pm 1.0$ & $21.1 \pm 0.3$ & $\begin{array}{l}< \\
0.001\end{array}$ & 0.464 & $\begin{array}{l}< \\
0.001\end{array}$ & 0.464 \\
\hline Lp (a)(mg/dl) & $16.0 \pm 1.1$ & $14.2 \pm 0.3$ & 0.140 & $20.8 \pm 1.9$ & $16.0 \pm 0.3$ & $\begin{array}{l}< \\
0.001\end{array}$ & 0.037 & $<.001$ & 0.037 \\
\hline
\end{tabular}




\begin{tabular}{|c|c|c|c|c|c|c|c|c|c|}
\hline & Men & & & Women & & & $\begin{array}{l}\text { Men vs } \\
\text { Women }\end{array}$ & & \\
\hline VAI & $1.9 \pm 0.1$ & $1.7 \pm 0.0$ & 0.082 & $2.8 \pm 0.1$ & $2.2 \pm 0.0$ & $\begin{array}{l}< \\
0.001\end{array}$ & $<0.001$ & $\begin{array}{l}< \\
0.001\end{array}$ & $<0.001$ \\
\hline LAP & $38.2 \pm 2.0$ & $33.2 \pm 0.6$ & 0.012 & $47.1 \pm 2.2$ & $36.2 \pm 0.5$ & $<.001$ & 0.003 & $\begin{array}{l}< \\
0.001\end{array}$ & 0.003 \\
\hline $\mathrm{UA}(\mathrm{mmol} / \mathrm{l})$ & $413.1 \pm 5.4$ & $341.2 \pm 1.4$ & $\begin{array}{l}< \\
0.001\end{array}$ & $347.1 \pm 4.8$ & $257.6 \pm 1.1$ & $\begin{array}{l}< \\
0.001\end{array}$ & $<0.001$ & $\begin{array}{l}< \\
0.001\end{array}$ & $<0.001$ \\
\hline WBC $\left(10^{9} / \mathrm{ml}\right)$ & $6.6 \pm 0.1$ & $6.5 \pm 0.0$ & 0.412 & $6.4 \pm 0.1$ & $6.0 \pm 0.0$ & $\begin{array}{l}< \\
0.001\end{array}$ & 0.267 & $\begin{array}{l}< \\
0.001\end{array}$ & $<0.001$ \\
\hline $\mathrm{Hb}(\mathrm{g} / \mathrm{dl})$ & $146.0 \pm 1.2$ & $152.3 \pm 0.3$ & $\begin{array}{l}< \\
0.001\end{array}$ & $127.5 \pm 1.1$ & $132.2 \pm 0.3$ & $\begin{array}{l}< \\
0.001\end{array}$ & $<0.001$ & $\begin{array}{l}< \\
0.001\end{array}$ & $<0.001$ \\
\hline Hs-CRP (mg/l) & $5.2 \pm 1.0$ & $2.5 \pm 0.1$ & $<.001$ & $4.6 \pm 0.6$ & $2.2 \pm 0.1$ & $<.001$ & 0.579 & 0.066 & 0.579 \\
\hline Ferrtin (ng/ml) & $181.6 \pm 11.7$ & $196.5 \pm 3.8$ & 0.264 & $125.8 \pm 7.5$ & $76.0 \pm 1.7$ & $\begin{array}{l}< \\
0.001\end{array}$ & $<0.001$ & $\begin{array}{l}< \\
0.001\end{array}$ & $<0.001$ \\
\hline
\end{tabular}

Data are mean \pm standard deviation (SD)

CKD, chronic kidney disease; non-CKD, non-chronic kidney disease; BMI, Body mass index; WC, waist circumference; SBP, systolic blood pressure; DBP, diastolic blood pressure; TC, total cholesterol; TG: triglycerides; LDL-C: low density lipoprotein cholesterol; HDLC: high density lipoprotein cholesterol; Apo B: apolipoprotein B; Lp (a), Lipoprotein (a);FPG, fasting plasma glucose; HOMA-IR: homeostasis model assessment of insulin resistance; TyG index: the product of triglycerides and fasting glucose; ALT, alanine aminotransferase; VAl: visceral adiposity index; LAP: lipid accumulation product; UA, uric acid; WBC, white blood cell count; Hs-CRP, hypersensitive C-reactive protein.

* $p$ value for difference between CKD and non-CKD participants

\& Interaction of sex by CKD status

Table 1 showed the non-adjusted mean values of CVD risk factors of CKD status in different genders. We can found an increase of CVD risk profile, such as TG, TC, LDL-C, TG/ HDL-C, Non-HDL-C, HOMA-IR, TyG, VAI, LAP and so on, in men and women with CKD in which compared with their non-CKD participants. Meanwhile, in CKD patients, we found that the TC, LDL-C, non-HDL-C and Apo B of women were significantly higher than men. Notably, between CKD patients and non-CKD people, women were more diverse than men in terms of WBC values. In addition, women with CKD have higher level of Lp(a), VAI and LAP compared with men with CKD. There was no difference of BMI between patients with CKD compared with their non-CKD ones. Besides, compared with non-CKD men, men with CKD had a lower level of ferritin, which was opposite to women. 
Table 2

Age- and BMl-adjusted mean levels of studied cardiovascular disease risk factors

\begin{tabular}{|c|c|c|c|c|c|c|c|c|c|}
\hline & Male & & & Female & & & $\begin{array}{l}\text { Male \& } \\
\text { Female }\end{array}$ & & \\
\hline & CKD & non-CKD & $\mathbf{P}^{*}$ & CKD & non-CKD & $\mathbf{P}^{*}$ & $\mathrm{P}(\mathrm{CKD})$ & $\begin{array}{l}\text { P(non- } \\
\text { CKD) }\end{array}$ & $\mathrm{P}$ (Interaction) \\
\hline $\mathbf{N}$ & 290 & 3399 & & 327 & 3983 & & & & \\
\hline $\mathrm{WC}(\mathrm{cm})$ & $84.1 \pm 0.4$ & $84.3 \pm 0.1$ & 0.730 & $80.0 \pm 0.4$ & $81.3 \pm 0.1$ & 0.001 & $<0.001$ & $\begin{array}{l}< \\
0.001\end{array}$ & $<0.001$ \\
\hline $\mathrm{SBP}(\mathrm{mmHg})$ & $130.2 \pm 1.0$ & $125.6 \pm 0.3$ & $\begin{array}{l}< \\
0.001\end{array}$ & $127.2 \pm 1.0$ & $123.3 \pm 0.3$ & $\begin{array}{l}< \\
0.001\end{array}$ & 0.798 & $\begin{array}{l}< \\
0.001\end{array}$ & 0.798 \\
\hline $\mathrm{DBP}(\mathrm{mmHg})$ & $82.5 \pm 0.6$ & $81.9 \pm 0.2$ & 0.328 & $78.0 \pm 0.6$ & $79.1 \pm 0.2$ & 0.078 & 0.001 & $\begin{array}{l}< \\
0.001\end{array}$ & 0.001 \\
\hline $\begin{array}{l}\text { eGFR(ml/min } \\
\left.\text { per1.73 } \mathrm{m}^{2}\right)\end{array}$ & $57.1 \pm 1.0$ & $83.6 \pm 0.3$ & $\begin{array}{l}< \\
0.001\end{array}$ & $58.6 \pm 0.7$ & $83.7 \pm 0.2$ & $\begin{array}{l}< \\
0.001\end{array}$ & 0.134 & 0.395 & 0.134 \\
\hline $\mathrm{TG}(\mathrm{mmol} / \mathrm{l})$ & $1.7 \pm 0.1$ & $1.6 \pm 0.0$ & 0.016 & $1.6 \pm 0.0$ & $1.5 \pm 0.0$ & 0.002 & 0.292 & $\begin{array}{l}< \\
0.001\end{array}$ & 0.292 \\
\hline TC(mmol/l) & $4.8 \pm 0.1$ & $4.8 \pm 0.0$ & 0.489 & $5.0 \pm 0.1$ & $4.9 \pm 0.0$ & 0.002 & $<0.001$ & $\begin{array}{l}< \\
0.001\end{array}$ & $<0.001$ \\
\hline HDL-C(mmol/l) & $1.3 \pm 0.0$ & $1.4 \pm 0.0$ & 0.016 & $1.5 \pm 0.0$ & $1.5 \pm 0.0$ & 0.481 & 0.001 & $\begin{array}{l}< \\
0.001\end{array}$ & 0.001 \\
\hline LDL-C(mmol/l) & $3.0 \pm 0.1$ & $3.0 \pm 0.0$ & 0.542 & $3.2 \pm 0.1$ & $3.0 \pm 0.0$ & 0.001 & $<0.001$ & 0.015 & $<0.001$ \\
\hline TG/HDL & $1.5 \pm 0.1$ & $1.3 \pm 0.0$ & 0.010 & $1.3 \pm 0.1$ & $1.1 \pm 0.0$ & 0.002 & 0.134 & $\begin{array}{l}< \\
0.001\end{array}$ & 0.631 \\
\hline $\begin{array}{l}\text { non- } \\
\text { HDL(mmol/l) }\end{array}$ & $3.5 \pm 0.1$ & $3.4 \pm 0.0$ & 0.101 & $3.6 \pm 0.1$ & $3.4 \pm 0.0$ & 0.001 & $<0.001$ & 0.417 & $<0.001$ \\
\hline Apo B(g/l) & $0.9 \pm 0.0$ & $0.9 \pm 0.0$ & 0.088 & $1.0 \pm 0.0$ & $0.9 \pm 0.0$ & $\begin{array}{l}< \\
0.001\end{array}$ & $<0.001$ & 0.331 & $<0.001$ \\
\hline ApoB/apo a1 & $0.9 \pm 0.0$ & $0.9 \pm 0.0$ & 0.156 & $0.9 \pm 0.0$ & $0.8 \pm 0.0$ & $\begin{array}{l}< \\
0.001\end{array}$ & 0.234 & $\begin{array}{l}< \\
0.001\end{array}$ & 0.234 \\
\hline FPG(mmol/l) & $5.6 \pm 0.1$ & $5.4 \pm 0.0$ & 0.003 & $5.7 \pm 0.1$ & $5.3 \pm 0.0$ & $\begin{array}{l}< \\
0.001\end{array}$ & 0.576 & $\begin{array}{l}< \\
0.001\end{array}$ & 0.576 \\
\hline $\begin{array}{l}\mathrm{HbA1c} \\
(\mathrm{mmol} / \mathrm{mol})\end{array}$ & $5.6 \pm 0.0$ & $5.6 \pm 0.0$ & 0.433 & $5.7 \pm 0.0$ & $5.6 \pm 0.0$ & 0.089 & 0.186 & 0.079 & 0.186 \\
\hline HOMA-IR & $6.4 \pm 0.4$ & $3.6 \pm 0.1$ & $\begin{array}{l}< \\
0.001\end{array}$ & $6.2 \pm 0.4$ & $3.4 \pm 0.1$ & $\begin{array}{l}< \\
0.001\end{array}$ & 0.913 & 0.147 & 0.913 \\
\hline Insulin & $1.1 \pm 0.0$ & $1.0 \pm 0.0$ & $<.001$ & $1.1 \pm 0.0$ & $1.0 \pm 0.0$ & $\begin{array}{l}< \\
0.001\end{array}$ & 0.933 & 0.455 & 0.933 \\
\hline TyG index & $8.8 \pm 0.0$ & $8.6 \pm 0.0$ & $\begin{array}{l}< \\
0.001\end{array}$ & $8.7 \pm 0.0$ & $8.5 \pm 0.0$ & $\begin{array}{l}< \\
0.001\end{array}$ & 0.161 & $\begin{array}{l}< \\
0.001\end{array}$ & 0.161 \\
\hline
\end{tabular}

Data are means $\pm S D$

CKD, chronic kidney disease; non-CKD, non-chronic kidney disease; BMI, Body mass index; WC, waist circumference; SBP, systolic blood pressure; DBP, diastolic blood pressure; TC, total cholesterol; TG: triglycerides; LDL-C: low density lipoprotein cholesterol; HDL-C: high density lipoprotein cholesterol; Apo B: apolipoprotein B; Lp (a), Lipoprotein (a);FPG, fasting plasma glucose; HOMAIR: homeostasis model assessment of insulin resistance; TyG index: the product of triglycerides and fasting glucose; ALT, alanine aminotransferase; VAl: visceral adiposity index; LAP: lipid accumulation product;. UA, uric acid; WBC, white blood cell count; Hs-CRP, hypersensitive C-reactive protein.

* $\mathrm{p}$ value for difference between CKD and non-CKD participants

\& Interaction of sex by CKD status 


\begin{tabular}{|c|c|c|c|c|c|c|c|c|c|}
\hline & Male & & & Female & & & $\begin{array}{l}\text { Male \& } \\
\text { Female }\end{array}$ & & \\
\hline ALT & $26.5 \pm 1.3$ & $27.4 \pm 0.4$ & 0.565 & $21.3 \pm 1.0$ & $21.1 \pm 0.3$ & 0.879 & 0.933 & $<.001$ & 0.458 \\
\hline $\mathrm{Lp}(\mathrm{a})(\mathrm{mg} / \mathrm{dl})$ & $14.8 \pm 1.2$ & $14.3 \pm 0.3$ & 0.668 & $19.2 \pm 1.3$ & $16.2 \pm 0.4$ & 0.027 & 0.029 & $\begin{array}{l}< \\
0.001\end{array}$ & 0.029 \\
\hline LAP & $36.2 \pm 1.6$ & $33.4 \pm 0.4$ & 0.085 & $39.8 \pm 1.5$ & $36.8 \pm 0.4$ & 0.063 & $<0.001$ & $\begin{array}{l}< \\
0.001\end{array}$ & $<0.001$ \\
\hline VAl & $1.9 \pm 0.1$ & $1.7 \pm 0.0$ & 0.022 & $2.5 \pm 0.1$ & $2.2 \pm 0.0$ & 0.003 & $<0.001$ & $\begin{array}{l}<.001 \\
0 .\end{array}$ & $<0.001$ \\
\hline $\mathrm{UA}(\mathrm{mmol} / \mathrm{l})$ & $422.1 \pm 5.0$ & $340.4 \pm 1.4$ & $\begin{array}{l}< \\
0.001\end{array}$ & $334.3 \pm 4.0$ & $258.7 \pm 1.1$ & $\begin{array}{l}< \\
0.001\end{array}$ & $<0.001$ & $<.001$ & $<0.001$ \\
\hline WBC $\left(10^{9} / \mathrm{ml}\right)$ & $6.7 \pm 0.1$ & $6.5 \pm 0.0$ & 0.049 & $6.6 \pm 0.1$ & $6.0 \pm 0.0$ & $\begin{array}{l}< \\
0.001\end{array}$ & 0.270 & $\begin{array}{l}< \\
0.001\end{array}$ & 0.270 \\
\hline $\mathrm{Hb}(\mathrm{g} / \mathrm{dl})$ & $149.7 \pm 1.1$ & $152.0 \pm 0.3$ & 0.042 & $127.7 \pm 1.0$ & $132.2 \pm 0.3$ & $\begin{array}{l}< \\
0.001\end{array}$ & $<0.001$ & $<.001$ & $<0.001$ \\
\hline Hs-CRP (mg/l) & $4.6 \pm 0.4$ & $2.6 \pm 0.1$ & $\begin{array}{l}< \\
0.001\end{array}$ & $3.8 \pm 0.5$ & $2.3 \pm 0.1$ & 0.006 & 0.598 & 0.069 & 0.598 \\
\hline Ferrtin (ng/ml) & $195.5 \pm 13.2$ & $195.3 \pm 3.7$ & 0.990 & $85.8 \pm 6.0$ & $79.3 \pm 1.6$ & 0.300 & $<0.001$ & $\begin{array}{l}< \\
0.001\end{array}$ & $<0.001$ \\
\hline \multicolumn{10}{|c|}{ Data are means $\pm S D$} \\
\hline \multicolumn{10}{|c|}{$\begin{array}{l}\text { CKD, chronic kidney disease; non-CKD, non-chronic kidney disease; BMI, Body mass index; WC, waist circumference; SBP, systolic } \\
\text { blood pressure; DBP, diastolic blood pressure; TC, total cholesterol; TG: triglycerides; LDL-C: Iow density lipoprotein cholesterol; } \\
\text { HDL-C: high density lipoprotein cholesterol; Apo B: apolipoprotein B; Lp (a), Lipoprotein (a);FPG, fasting plasma glucose; HOMA- } \\
\text { IR: homeostasis model assessment of insulin resistance; TyG index: the product of triglycerides and fasting glucose; ALT, } \\
\text { alanine aminotransferase; VAl: visceral adiposity index; LAP: lipid accumulation product;. UA, uric acid; WBC, white blood cell } \\
\text { count; Hs-CRP, hypersensitive C-reactive protein. }\end{array}$} \\
\hline \multicolumn{10}{|c|}{ * $\mathrm{p}$ value for difference between CKD and non-CKD participants } \\
\hline \multicolumn{10}{|c|}{ \& Interaction of sex by CKD status } \\
\hline
\end{tabular}


Table 3

Age-, BMI-, and HOMA-IR-adjusted mean levels of studied cardiovascular disease risk factors

\begin{tabular}{|c|c|c|c|c|c|c|c|c|c|}
\hline & Male & & & Female & & & M\&F & & \\
\hline & CKD & non-CKD & $\mathbf{P}^{*}$ & CKD & non-CKD & $\mathbf{P}^{*}$ & $\mathrm{P}(\mathrm{CKD})$ & $\begin{array}{l}\mathrm{P}(\text { non- } \\
\text { CKD) }\end{array}$ & $\mathrm{P}(\text { Interaction })^{\&}$ \\
\hline $\mathbf{N}$ & 290 & 3399 & & 327 & 3987 & & & & \\
\hline WC(cm) & $84.0 \pm 0.4$ & $84.3 \pm 0.1$ & 0.534 & $79.9 \pm 0.4$ & $81.3 \pm 0.1$ & $\begin{array}{l}< \\
0.001\end{array}$ & $<0.001$ & $\begin{array}{l}<.001 \\
0.00\end{array}$ & $<0.001$ \\
\hline SBP(mmHg) & $130.1 \pm 1.0$ & $125.6 \pm 0.3$ & $\begin{array}{l}< \\
0.001\end{array}$ & $126.9 \pm 1.0$ & $123.4 \pm 0.3$ & 0.001 & 0.797 & $\begin{array}{l}<.001 \\
0.00\end{array}$ & $<0.001$ \\
\hline DBP(mmHg) & $82.6 \pm 0.6$ & $81.9 \pm 0.2$ & 0.295 & $78.0 \pm 0.6$ & $79.1 \pm 0.2$ & 0.060 & 0.001 & $\begin{array}{l}< \\
0.001\end{array}$ & $<0.001$ \\
\hline $\begin{array}{l}\text { eGFR(ml/min } \\
\left.\text { per1.73 } \mathrm{m}^{2}\right)\end{array}$ & $57.2 \pm 1.0$ & $83.6 \pm 0.3$ & $<.001$ & $58.7 \pm 0.7$ & $83.7 \pm 0.2$ & $<0.001$ & 0.135 & 0.405 & 0.539 \\
\hline $\mathrm{TG}(\mathrm{mmol} / \mathrm{l})$ & $1.7 \pm 0.1$ & $1.6 \pm 0.0$ & 0.121 & $1.6 \pm 0.0$ & $1.5 \pm 0.0$ & 0.019 & 0.279 & $\begin{array}{l}< \\
0.001\end{array}$ & $<0.001$ \\
\hline $\mathrm{TC}(\mathrm{mmol} / \mathrm{l})$ & $4.8 \pm 0.1$ & $4.8 \pm 0.0$ & 0.420 & $5.0 \pm 0.1$ & $4.9 \pm 0.0$ & 0.003 & $<0.001$ & $\dot{0} 001$ & $<0.001$ \\
\hline $\begin{array}{l}\text { HDL- } \\
\mathrm{C}(\mathrm{mmol} / \mathrm{l})\end{array}$ & $1.3 \pm 0.0$ & $1.4 \pm 0.0$ & 0.058 & $1.5 \pm 0.0$ & $1.5 \pm 0.0$ & 0.827 & 0.001 & $\begin{array}{l}< \\
0.001\end{array}$ & $<0.001$ \\
\hline $\begin{array}{l}\text { LDL- } \\
\text { C(mmol/I) }\end{array}$ & $3.0 \pm 0.1$ & $3.0 \pm 0.0$ & 0.402 & $3.2 \pm 0.1$ & $3.0 \pm 0.0$ & 0.002 & $<0.001$ & 0.015 & $<0.001$ \\
\hline TG/HDL & $1.4 \pm 0.1$ & $1.3 \pm 0.0$ & 0.091 & $1.2 \pm 0.1$ & $1.1 \pm 0.0$ & 0.018 & 0.640 & $\begin{array}{l}<.001 \\
0.00\end{array}$ & $<0.001$ \\
\hline $\begin{array}{l}\text { non- } \\
\text { HDL(mmol/l) }\end{array}$ & $3.5 \pm 0.1$ & $3.4 \pm 0.0$ & 0.120 & $3.6 \pm 0.1$ & $3.4 \pm 0.0$ & 0.002 & $<0.001$ & 0.444 & 0.579 \\
\hline Apo B(g/l) & $0.9 \pm 0.0$ & $0.9 \pm 0.0$ & 0.093 & $1.0 \pm 0.0$ & $0.9 \pm 0.0$ & $\begin{array}{l}< \\
0.001\end{array}$ & $<0.001$ & 0.355 & 0.657 \\
\hline ApoB/apo a1 & $0.9 \pm 0.0$ & $0.9 \pm 0.0$ & 0.239 & $0.9 \pm 0.0$ & $0.8 \pm 0.0$ & 0.002 & 0.231 & $\hat{0} .001$ & $<0.001$ \\
\hline FPG(mmol/l) & $5.4 \pm 0.1$ & $5.4 \pm 0.0$ & 0.631 & $5.6 \pm 0.1$ & $5.3 \pm 0.0$ & $\begin{array}{l}< \\
0.001\end{array}$ & 0.498 & 0.001 & 0.005 \\
\hline $\begin{array}{l}\mathrm{HbA} 1 \mathrm{c} \\
(\mathrm{mmol} / \mathrm{mol})\end{array}$ & $5.5 \pm 0.1$ & $5.6 \pm 0.0$ & 0.068 & $5.6 \pm 0.0$ & $5.6 \pm 0.0$ & 0.604 & 0.167 & 0.123 & 0.298 \\
\hline Insulin & $1.1 \pm 0.0$ & $1.0 \pm 0.0$ & $\begin{array}{l}< \\
0.001\end{array}$ & $1.1 \pm 0.0$ & $1.0 \pm 0.0$ & 0.012 & 0.990 & 0.040 & 0.071 \\
\hline Tyg index & $8.7 \pm 0.0$ & $8.6 \pm 0.0$ & 0.017 & $8.6 \pm 0.0$ & $8.6 \pm 0.0$ & 0.006 & 0.135 & $\begin{array}{l}< \\
0.001\end{array}$ & $<0.001$ \\
\hline
\end{tabular}

Data are means $\pm S D$

BMI: body mass index; HOMA-IR: homeostasis model assessment of insulin resistance; CKD, chronic kidney disease; non-CKD, non-chronic kidney disease; BMI, Body mass index; WC, waist circumference; SBP, systolic blood pressure; DBP, diastolic blood pressure; TC, total cholesterol; TG: triglycerides; LDL-C: low density lipoprotein cholesterol; HDL-C: high density lipoprotein cholesterol; Apo B: apolipoprotein B; Lp (a), Lipoprotein (a);FPG, fasting plasma glucose; TyG index: the product of triglycerides and fasting glucose; ALT, alanine aminotransferase; VAl: visceral adiposity index; LAP: lipid accumulation product;. UA, uric acid; WBC, white blood cell count; Hs-CRP, hypersensitive C-reactive protein.

* $p$ value for difference between CKD and non-CKD participants

\& Interaction of sex by CKD status 


\begin{tabular}{|c|c|c|c|c|c|c|c|c|c|}
\hline & Male & & & Female & & & M\&F & & \\
\hline ALT & $26.3 \pm 1.3$ & $27.4 \pm 0.4$ & 0.464 & $21.1 \pm 1.0$ & $21.1 \pm 0.3$ & 0.971 & 0.460 & $\begin{array}{l}< \\
0.001\end{array}$ & $<0.001$ \\
\hline $\begin{array}{l}\operatorname{Lp}(a) \\
(\mathrm{mg} / \mathrm{dl})\end{array}$ & $14.9 \pm 1.2$ & $14.3 \pm 0.3$ & 0.636 & $19.1 \pm 1.3$ & $16.2 \pm 0.4$ & 0.036 & 0.029 & $\begin{array}{l}<.001 \\
0\end{array}$ & $<0.001$ \\
\hline LAP & $34.9 \pm 1.6$ & $33.5 \pm 0.4$ & 0.380 & $38.5 \pm 1.5$ & $36.9 \pm 0.4$ & 0.320 & $<0.001$ & $<.001$ & $<0.001$ \\
\hline VAI & $1.8 \pm 0.1$ & $1.7 \pm 0.0$ & 0.165 & $2.4 \pm 0.1$ & $2.2 \pm 0.0$ & 0.032 & $<0.001$ & $\dot{0} 001$ & $<0.001$ \\
\hline UA(mmol/l) & $421.4 \pm 5.1$ & $340.5 \pm 1.4$ & $\begin{array}{l}< \\
0.001\end{array}$ & $332.7 \pm 4.1$ & $258.8 \pm 1.1$ & $\begin{array}{l}<.001 \\
0.001\end{array}$ & $<0.001$ & $\begin{array}{l}<.001 \\
0\end{array}$ & $<0.001$ \\
\hline $\begin{array}{l}\text { WBC } \\
\left(10^{9} / \mathrm{ml}\right)\end{array}$ & $6.7 \pm 0.1$ & $6.5 \pm 0.0$ & 0.088 & $6.5 \pm 0.1$ & $6.0 \pm 0.0$ & $<_{0.001}$ & 0.273 & $\stackrel{<}{0.001}$ & $<0.001$ \\
\hline $\mathrm{Hb}(\mathrm{g} / \mathrm{dl})$ & $149.4 \pm 1.1$ & $152.0 \pm 0.3$ & 0.024 & $127.4 \pm 1.0$ & $132.2 \pm 0.3$ & $\begin{array}{l}< \\
0.001\end{array}$ & $<0.001$ & $<.001$ & $<0.001$ \\
\hline $\begin{array}{l}\text { Hs-CRP } \\
(\mathrm{mg} / \mathrm{l})\end{array}$ & $4.5 \pm 0.5$ & $2.6 \pm 0.1$ & $\begin{array}{l}< \\
0.001\end{array}$ & $3.5 \pm 0.5$ & $2.3 \pm 0.1$ & 0.024 & 0.603 & 0.078 & 0.069 \\
\hline $\begin{array}{l}\text { Ferrtin } \\
\text { (ng/ml) }\end{array}$ & $193.3 \pm 13.3$ & $195.5 \pm 3.7$ & 0.873 & $83.5 \pm 6.0$ & $79.5 \pm 1.6$ & 0.531 & $<0.001$ & $\begin{array}{l}<.001 \\
0.00\end{array}$ & $<0.001$ \\
\hline \multicolumn{10}{|c|}{ Data are means $\pm S D$} \\
\hline \multicolumn{10}{|c|}{$\begin{array}{l}\text { BMI: body mass index; HOMA-IR: homeostasis model assessment of insulin resistance; CKD, chronic kidney disease; non-CKD, } \\
\text { non-chronic kidney disease; BMI, Body mass index; WC, waist circumference; SBP, systolic blood pressure; DBP, diastolic blood } \\
\text { pressure; TC, total cholesterol; TG: triglycerides; LDL-C: low density lipoprotein cholesterol; HDL-C: high density lipoprotein } \\
\text { cholesterol; Apo B: apolipoprotein B; Lp (a), Lipoprotein (a);FPG, fasting plasma glucose; TyG index: the product of triglycerides } \\
\text { and fasting glucose; ALT, alanine aminotransferase; VAl: visceral adiposity index; LAP: lipid accumulation product;. UA, uric acid } \\
\text { WBC, white blood cell count; Hs-CRP, hypersensitive C-reactive protein. }\end{array}$} \\
\hline \multicolumn{10}{|c|}{ * $p$ value for difference between CKD and non-CKD participants } \\
\hline \multicolumn{10}{|c|}{ \& Interaction of sex by CKD status } \\
\hline
\end{tabular}

Since CKD is related with age and CVD is related with BMI, then we adjusted age and BMI in Table 2. As the table showed, the mean levels of TC, LDL-C, Non-HDL-C and Apo B of women with CKD were greater than men with CKD under the adjusted of age and BMI. Besides, the differences of WBC between women with CKD and non-CKD ones were more striking than men. Moreover, compared with men with CKD, women with CKD have higher level of Lp(a), VAI and LAP. However, there was little statistical evidence about sex heterogeneity in the association of CKD with TG, TG/HDL, Apo B/apo a1, FPG, HbA1c, HOMA-IR, TyG, ALT and hs-CRP. Also, we observed some interactions between sex with CKD status with statistically significant in some CVD risk factors, such as TC, HDL-C, LDL-C, non-HDL-C, Lp (a), LAP and VAI(all $p$ values $<0.05$ ). After further adjustment for HOMA-IR, there was a little change between women with CKD and men with CKD about the difference of these risk factors (Table 3). 
Table 4

Age- and VAl-adjusted mean levels of studied cardiovascular disease risk factors

\begin{tabular}{|c|c|c|c|c|c|c|c|c|c|}
\hline & Male & & & Female & & & M\&F & & \\
\hline & CKD & non-CKD & $\mathbf{P}^{*}$ & CKD & non-CKD & $\mathrm{P}^{*}$ & $P(C K D)$ & $\begin{array}{l}\text { P(non- } \\
\text { CKD) }\end{array}$ & $\mathrm{P}(\text { Interaction })^{\&}$ \\
\hline $\mathbf{N}$ & 290 & 3399 & & 327 & 3987 & & & & \\
\hline $\mathrm{BMI}\left(\mathrm{kg} / \mathrm{m}^{2}\right)$ & $23.3 \pm 0.2$ & $23.3 \pm 0.1$ & 0.701 & $22.9 \pm 0.2$ & $23.4 \pm 0.1$ & 0.012 & 0.082 & 0.005 & 0.001 \\
\hline WC(cm) & $84.2 \pm 0.6$ & $84.3 \pm 0.2$ & 0.844 & $78.9 \pm 0.5$ & $81.4 \pm 0.1$ & $\begin{array}{l}< \\
0.001\end{array}$ & $<0.001$ & $\begin{array}{l}< \\
0.001\end{array}$ & $<0.001$ \\
\hline $\mathrm{SBP}(\mathrm{mmHg})$ & $130.3 \pm 1.0$ & $125.6 \pm 0.3$ & $<.001$ & $126.4 \pm 1.0$ & $123.4 \pm 0.3$ & 0.004 & 0.358 & $\begin{array}{l}< \\
0.001\end{array}$ & $<0.001$ \\
\hline $\mathrm{DBP}(\mathrm{mmHg})$ & $82.5 \pm 0.7$ & $81.9 \pm 0.2$ & 0.331 & $77.5 \pm 0.6$ & $79.2 \pm 0.2$ & 0.012 & $<0.001$ & $\begin{array}{l}<.001 \\
0.001\end{array}$ & $<0.001$ \\
\hline $\begin{array}{l}\text { eGFR }(\mathrm{ml} / \mathrm{min} \\
\left.\text { per1.73 } \mathrm{m}^{2}\right)\end{array}$ & $57.2 \pm 1.0$ & $83.6 \pm 0.3$ & $<.001$ & $58.6 \pm 0.7$ & $83.7 \pm 0.2$ & $<.001$ & 0.122 & 0.259 & 0.244 \\
\hline $\mathrm{TG}(\mathrm{mmol} / \mathrm{l})$ & $1.6 \pm 0.0$ & $1.6 \pm 0.0$ & 0.418 & $1.5 \pm 0.0$ & $1.5 \pm 0.0$ & 0.206 & $<0.001$ & $\begin{array}{l}< \\
0.001\end{array}$ & $<0.001$ \\
\hline $\mathrm{TC}(\mathrm{mmol} / \mathrm{l})$ & $4.8 \pm 0.1$ & $4.8 \pm 0.0$ & 0.590 & $5.0 \pm 0.1$ & $4.9 \pm 0.0$ & 0.010 & $<0.001$ & 0.014 & $<0.001$ \\
\hline $\mathrm{HDL}-\mathrm{C}(\mathrm{mmol} / \mathrm{l})$ & $1.3 \pm 0.0$ & $1.4 \pm 0.0$ & 0.139 & $1.5 \pm 0.0$ & $1.5 \pm 0.0$ & 0.251 & $<0.001$ & $\begin{array}{l}<.001 \\
0.00\end{array}$ & $<0.001$ \\
\hline LDL-C(mmol/l) & $3.0 \pm 0.1$ & $3.0 \pm 0.0$ & 0.358 & $3.2 \pm 0.1$ & $3.0 \pm 0.0$ & 0.002 & $<0.001$ & 0.003 & $<0.001$ \\
\hline TG/HDL & $1.3 \pm 0.0$ & $1.3 \pm 0.0$ & 0.124 & $1.1 \pm 0.0$ & $1.1 \pm 0.0$ & 0.127 & $<0.001$ & $<.001$ & $<0.001$ \\
\hline $\begin{array}{l}\text { non- } \\
\text { HDL(mmol/l) }\end{array}$ & $3.5 \pm 0.1$ & $3.4 \pm 0.0$ & 0.283 & $3.5 \pm 0.1$ & $3.4 \pm 0.0$ & 0.016 & 0.016 & $\begin{array}{l}<.001 \\
0.001\end{array}$ & $<0.001$ \\
\hline Apo B(g/l) & $0.9 \pm 0.0$ & $0.9 \pm 0.0$ & 0.178 & $1.0 \pm 0.0$ & $0.9 \pm 0.0$ & $\begin{array}{l}< \\
0.001\end{array}$ & 0.012 & $\begin{array}{l}< \\
0.001\end{array}$ & 0.004 \\
\hline ApoB/apo a1 & $0.9 \pm 0.0$ & $0.9 \pm 0.0$ & 0.342 & $0.8 \pm 0.0$ & $0.8 \pm 0.0$ & 0.014 & 0.215 & $<.001$ & $<0.001$ \\
\hline $\mathrm{FPG}(\mathrm{mmol} / \mathrm{l})$ & $5.6 \pm 0.1$ & $5.4 \pm 0.0$ & 0.009 & $5.7 \pm 0.1$ & $5.3 \pm 0.0$ & $<.001$ & 0.362 & $\begin{array}{l}< \\
0.001\end{array}$ & $<0.001$ \\
\hline $\begin{array}{l}\mathrm{HbA} 1 \mathrm{c} \\
(\mathrm{mmol} / \mathrm{mol})\end{array}$ & $5.6 \pm 0.0$ & $5.6 \pm 0.0$ & 0.359 & $5.7 \pm 0.1$ & $5.6 \pm 0.0$ & 0.293 & 0.779 & 0.002 & 0.004 \\
\hline Insulin & $1.1 \pm 0.0$ & $1.0 \pm 0.0$ & $<.001$ & $1.1 \pm 0.0$ & $1.0 \pm 0.0$ & $<.001$ & 0.067 & 0.060 & 0.017 \\
\hline HOMA-IR & $6.3 \pm 0.4$ & $3.6 \pm 0.1$ & $\begin{array}{l}< \\
0.001\end{array}$ & $6.0 \pm 0.4$ & $3.4 \pm 0.0$ & $\begin{array}{l}< \\
0.001\end{array}$ & 0.228 & 0.002 & 0.001 \\
\hline
\end{tabular}

Data are means $\pm S D$

VAl: visceral adiposity index; CKD, chronic kidney disease; non-CKD, non-chronic kidney disease; BMI, Body mass index; WC, waist circumference; SBP, systolic blood pressure; DBP, diastolic blood pressure; TC, total cholesterol; TG: triglycerides; LDL-C: low density lipoprotein cholesterol; HDL-C: high density lipoprotein cholesterol; Apo B: apolipoprotein B; Lp (a), Lipoprotein (a); FPG, fasting plasma glucose; HOMA-IR: homeostasis model assessment of insulin resistance; TyG index: the product of triglycerides and fasting glucose; ALT, alanine aminotransferase; LAP: lipid accumulation product;: UA, uric acid; WBC, white blood cell count; Hs-CRP, hypersensitive C-reactive protein.

* $p$ value for difference between CKD and non-CKD participants

\& Interaction of sex by CKD status 


\begin{tabular}{|c|c|c|c|c|c|c|c|c|c|}
\hline & Male & & & Female & & & M\&F & & \\
\hline Tyg index & $8.7 \pm 0.0$ & $8.6 \pm 0.0$ & 0.001 & $8.6 \pm 0.0$ & $8.6 \pm 0.0$ & 0.007 & $<0.001$ & $\begin{array}{l}< \\
0.001\end{array}$ & $<0.001$ \\
\hline ALT & $26.3 \pm 1.3$ & $27.4 \pm 0.4$ & 0.439 & $20.7 \pm 1.0$ & $21.1 \pm 0.3$ & 0.679 & 0.090 & $\begin{array}{l}<.001 \\
0.00\end{array}$ & $<0.001$ \\
\hline LAP & $33.6 \pm 1.1$ & $33.6 \pm 0.3$ & 0.990 & $34.9 \pm 1.0$ & $37.2 \pm 0.3$ & 0.023 & 0.003 & $\begin{array}{l}< \\
0.001\end{array}$ & $<0.001$ \\
\hline $\mathrm{Lp}(\mathrm{a})(\mathrm{mg} / \mathrm{dl})$ & $15.0 \pm 1.2$ & $14.3 \pm 0.3$ & 0.580 & $19.4 \pm 1.3$ & $16.1 \pm 0.4$ & 0.020 & 0.009 & $\begin{array}{l}< \\
0.001\end{array}$ & $<0.001$ \\
\hline $\mathrm{UA}(\mathrm{mmol} / \mathrm{l})$ & $418.5 \pm 4.8$ & $340.7 \pm 1.3$ & $\begin{array}{l}< \\
0.001\end{array}$ & $329.7 \pm 3.8$ & $259.1 \pm 1.0$ & $\begin{array}{l}< \\
0.001\end{array}$ & $<0.001$ & $\begin{array}{l}<.001 \\
0.00\end{array}$ & $<0.001$ \\
\hline WBC $\left(10^{9} / \mathrm{ml}\right)$ & $6.7 \pm 0.1$ & $6.5 \pm 0.0$ & 0.080 & $6.5 \pm 0.1$ & $6.0 \pm 0.0$ & $\begin{array}{l}< \\
0.001\end{array}$ & 0.087 & $\begin{array}{l}< \\
0.001\end{array}$ & $<0.001$ \\
\hline $\mathrm{Hb}(\mathrm{g} / \mathrm{dl})$ & $149.6 \pm 1.1$ & $152.0 \pm 0.3$ & 0.034 & $127.3 \pm 1.0$ & $132.2 \pm 0.3$ & $\begin{array}{l}< \\
0.001\end{array}$ & $<0.001$ & $\begin{array}{l}<.001 \\
0.00\end{array}$ & $<0.001$ \\
\hline Hs-CRP (mg/l) & $4.6 \pm 0.5$ & $2.6 \pm 0.1$ & $\begin{array}{l}<.001 \\
0.001\end{array}$ & $3.7 \pm 0.5$ & $2.3 \pm 0.1$ & 0.009 & 0.537 & 0.028 & 0.021 \\
\hline Ferrtin (ng/ml) & $190.4 \pm 13.0$ & $195.7 \pm 3.7$ & 0.694 & $82.9 \pm 6.0$ & $79.6 \pm 1.6$ & 0.594 & $<0.001$ & $\begin{array}{l}< \\
0.001\end{array}$ & $<0.001$ \\
\hline \multicolumn{10}{|c|}{ Data are means $\pm S D$} \\
\hline \multicolumn{10}{|c|}{$\begin{array}{l}\text { VAl: visceral adiposity index; CKD, chronic kidney disease; non-CKD, non-chronic kidney disease; BMI, Body mass index; WC, } \\
\text { waist circumference; SBP, systolic blood pressure; DBP, diastolic blood pressure; TC, total cholesterol; TG: triglycerides; LDL-C: } \\
\text { low density lipoprotein cholesterol; HDL-C: high density lipoprotein cholesterol; Apo B: apolipoprotein B; Lp (a), Lipoprotein (a) } \\
\text { FPG, fasting plasma glucose; HOMA-IR: homeostasis model assessment of insulin resistance; TyG index: the product of } \\
\text { triglycerides and fasting glucose; ALT, alanine aminotransferase; LAP: lipid accumulation product;. UA, uric acid; WBC, white } \\
\text { blood cell count; Hs-CRP, hypersensitive C-reactive protein. }\end{array}$} \\
\hline \multicolumn{10}{|c|}{ * $\mathrm{p}$ value for difference between CKD and non-CKD participants } \\
\hline \multicolumn{10}{|c|}{ \& Interaction of sex by CKD status } \\
\hline
\end{tabular}


Table 5

Age-, VAl-, and HOMA-IR- adjusted mean levels of studied cardiovascular disease risk factors

\begin{tabular}{|c|c|c|c|c|c|c|c|c|c|}
\hline & Male & & & Female & & & M\&F & & \\
\hline & CKD & non-CKD & $\mathbf{P}^{*}$ & CKD & non-CKD & $\mathbf{P}^{*}$ & $P(C K D)$ & $\begin{array}{l}\text { P(non- } \\
\text { CKD) }\end{array}$ & $\mathrm{P}(\text { Interaction })^{\&}$ \\
\hline $\mathbf{N}$ & 290 & 3399 & & 327 & 3987 & & & & \\
\hline $\mathrm{BMI}\left(\mathrm{kg} / \mathrm{m}^{2}\right)$ & $23.2 \pm 0.2$ & $23.3 \pm 0.1$ & 0.875 & $22.8 \pm 0.2$ & $23.4 \pm 0.1$ & 0.004 & 0.108 & 0.010 & 0.003 \\
\hline WC(cm) & $83.9 \pm 0.6$ & $84.3 \pm 0.2$ & 0.493 & $78.7 \pm 0.5$ & $81.4 \pm 0.1$ & 0.000 & $<0.001$ & $<.001$ & $<0.001$ \\
\hline $\mathrm{SBP}(\mathrm{mmHg})$ & $130.1 \pm 1.0$ & $125.6 \pm 0.3$ & $\begin{array}{l}< \\
0.001\end{array}$ & $126.2 \pm 1.0$ & $123.4 \pm 0.3$ & 0.009 & 0.357 & < 0.001 & $<0.001$ \\
\hline $\mathrm{DBP}(\mathrm{mmHg})$ & $82.5 \pm 0.7$ & $81.9 \pm 0.2$ & 0.361 & $77.4 \pm 0.6$ & $79.2 \pm 0.2$ & 0.008 & $<0.001$ & $<.001$ & $<0.001$ \\
\hline $\begin{array}{l}\text { eGFR }(\mathrm{ml} / \mathrm{min} \\
\left.\text { per1.73 } \mathrm{m}^{2}\right)\end{array}$ & $57.2 \pm 1.0$ & $83.5 \pm 0.3$ & $\dot{0}_{0.001}$ & $58.7 \pm 0.7$ & $83.7 \pm 0.2$ & $\dot{0}_{0.001}$ & 0.129 & 0.276 & 0.312 \\
\hline $\mathrm{TC}(\mathrm{mmol} / \mathrm{l})$ & $4.8 \pm 0.1$ & $4.8 \pm 0.0$ & 0.521 & $5.0 \pm 0.1$ & $4.9 \pm 0.0$ & 0.013 & $<0.001$ & 0.014 & $<0.001$ \\
\hline $\mathrm{TG}(\mathrm{mmol} / \mathrm{l})$ & $1.6 \pm 0.0$ & $1.6 \pm 0.0$ & 0.469 & $1.5 \pm 0.0$ & $1.5 \pm 0.0$ & 0.228 & $<0.001$ & $\begin{array}{l}< \\
0.001\end{array}$ & $<0.001$ \\
\hline LDL-C(mmol/l) & $3.0 \pm 0.1$ & $3.0 \pm 0.0$ & 0.350 & $3.2 \pm 0.1$ & $3.0 \pm 0.0$ & 0.005 & $<0.001$ & 0.002 & $<0.001$ \\
\hline HDL-C(mmol/l) & $1.3 \pm 0.0$ & $1.4 \pm 0.0$ & 0.178 & $1.5 \pm 0.0$ & $1.5 \pm 0.0$ & 0.222 & $<0.001$ & ¿. 001 & $<0.001$ \\
\hline TG/HDL & $1.3 \pm 0.0$ & $1.3 \pm 0.0$ & 0.140 & $1.2 \pm 0.0$ & $1.1 \pm 0.0$ & 0.081 & $<0.001$ & $<.001$ & $<0.001$ \\
\hline $\begin{array}{l}\text { non- } \\
\text { HDL(mmol/l) }\end{array}$ & $3.5 \pm 0.1$ & $3.4 \pm 0.0$ & 0.256 & $3.5 \pm 0.0$ & $3.4 \pm 0.0$ & 0.021 & 0.016 & $<.001$ & $<0.001$ \\
\hline Apo B(g/l) & $0.9 \pm 0.0$ & $0.9 \pm 0.0$ & 0.170 & $1.0 \pm 0.0$ & $0.9 \pm 0.0$ & 0.001 & 0.011 & $<.001$ & 0.005 \\
\hline ApoB/apo a1 & $0.9 \pm 0.0$ & $0.9 \pm 0.0$ & 0.404 & $0.8 \pm 0.0$ & $0.8 \pm 0.0$ & 0.027 & 0.220 & $<.001$ & $<0.001$ \\
\hline $\mathrm{FPG}(\mathrm{mmol} / \mathrm{l})$ & $5.4 \pm 0.1$ & $5.4 \pm 0.0$ & 0.781 & $5.5 \pm 0.1$ & $5.3 \pm 0.0$ & 0.001 & 0.664 & $<.001$ & $<0.001$ \\
\hline $\begin{array}{l}\mathrm{HbA} 1 \mathrm{c} \\
(\mathrm{mmol} / \mathrm{mol})\end{array}$ & $5.5 \pm 0.0$ & $5.6 \pm 0.0$ & 0.055 & $5.6 \pm 0.0$ & $5.6 \pm 0.0$ & 0.971 & 0.561 & 0.008 & 0.019 \\
\hline Insulin & $1.1 \pm 0.0$ & $1.0 \pm 0.0$ & 0.001 & $1.1 \pm 0.0$ & $1.0 \pm 0.0$ & 0.098 & 0.167 & 0.986 & 0.559 \\
\hline Tyg index & $8.7 \pm 0.0$ & $8.6 \pm 0.0$ & 0.043 & $8.6 \pm 0.0$ & $8.6 \pm 0.0$ & 0.118 & $<0.001$ & $\begin{array}{l}< \\
0.001\end{array}$ & $<0.001$ \\
\hline ALT & $26.1 \pm 1.4$ & $27.4 \pm 0.4$ & 0.366 & $20.6 \pm 1.0$ & $21.2 \pm 0.3$ & 0.588 & 0.096 & $<.001$ & $<0.001$ \\
\hline
\end{tabular}

Data are means $\pm S D$

VAI: visceral adiposity index; HOMA-IR: homeostasis model assessment of insulin resistance; CKD, chronic kidney disease; nonCKD, non-chronic kidney disease; BMI, Body mass index; WC, waist circumference; SBP, systolic blood pressure; DBP, diastolic blood pressure; TC, total cholesterol; TG: triglycerides; LDL-C: low density lipoprotein cholesterol; HDL-C: high density lipoprotein cholesterol; Apo B: apolipoprotein B; Lp(a), Lipoprotein (a); FPG, fasting plasma glucose; TyG index: the product of triglycerides and fasting glucose; ALT, alanine aminotransferase; LAP: lipid accumulation product;. UA, uric acid; WBC, white blood cell count; Hs-CRP, hypersensitive C-reactive protein.

* $p$ value for difference between CKD and non-CKD participants

\& Interaction of sex by CKD status 


\begin{tabular}{|c|c|c|c|c|c|c|c|c|c|}
\hline & Male & & & Female & & & M\&F & & \\
\hline LAP & $33.1 \pm 1.1$ & $33.6 \pm 0.3$ & 0.638 & $34.4 \pm 1.0$ & $37.2 \pm 0.3$ & 0.005 & 0.005 & $\begin{array}{l}< \\
0.001\end{array}$ & $<0.001$ \\
\hline Lp (a)(mg/dl) & $15.0 \pm 1.2$ & $14.3 \pm 0.3$ & 0.568 & $19.2 \pm 1.3$ & $16.2 \pm 0.4$ & 0.027 & 0.008 & $\begin{array}{l}<.001 \\
0.00\end{array}$ & $<0.001$ \\
\hline $\mathrm{UA}(\mathrm{mmol} / \mathrm{l})$ & $419.0 \pm 4.8$ & $340.7 \pm 1.3$ & $\begin{array}{l}< \\
0.001\end{array}$ & $329.1 \pm 3.8$ & $259.1 \pm 1.0$ & $\begin{array}{l}< \\
0.001\end{array}$ & $<0.001$ & $\begin{array}{l}< \\
0.001\end{array}$ & $<0.001$ \\
\hline WBC $\left(10^{9} / \mathrm{ml}\right)$ & $6.7 \pm 0.1$ & $6.5 \pm 0.0$ & 0.117 & $6.5 \pm 0.1$ & $6.0 \pm 0.0$ & $<.001$ & 0.110 & $\begin{array}{l}<.001 \\
0.00\end{array}$ & $<0.001$ \\
\hline $\mathrm{Hb}(\mathrm{g} / \mathrm{dl})$ & $149.3 \pm 1.1$ & $152.0 \pm 0.3$ & 0.019 & $127.0 \pm 1.0$ & $132.2 \pm 0.3$ & $\begin{array}{l}< \\
0.001\end{array}$ & $<0.001$ & $\begin{array}{l}<.001 \\
0.00\end{array}$ & $<0.001$ \\
\hline Hs-CRP (mg/l) & $4.5 \pm 0.5$ & $2.6 \pm 0.1$ & $\dot{0}_{0.001}$ & $3.5 \pm 0.5$ & $2.3 \pm 0.1$ & 0.031 & 0.600 & 0.037 & 0.032 \\
\hline Ferrtin (ng/ml) & $189.7 \pm 13.1$ & $195.8 \pm 3.7$ & 0.656 & $81.2 \pm 6.0$ & $79.7 \pm 1.6$ & 0.805 & $<0.001$ & $\begin{array}{l}<.001 \\
0.00\end{array}$ & $<0.001$ \\
\hline \multicolumn{10}{|c|}{ Data are means $\pm S D$} \\
\hline \multicolumn{10}{|c|}{$\begin{array}{l}\text { VAl: visceral adiposity index; HOMA-IR: homeostasis model assessment of insulin resistance; CKD, chronic kidney disease; non- } \\
\text { CKD, non-chronic kidney disease; BMI, Body mass index; WC, waist circumference; SBP, systolic blood pressure; DBP, diastolic } \\
\text { blood pressure; TC, total cholesterol; TG: triglycerides; LDL-C: low density lipoprotein cholesterol; HDL-C: high density lipoprotein } \\
\text { cholesterol; Apo B: apolipoprotein B; Lp(a), Lipoprotein (a); FPG, fasting plasma glucose; TyG index: the product of triglycerides } \\
\text { and fasting glucose; ALT, alanine aminotransferase; LAP: lipid accumulation product,. UA, uric acid; WBC, white blood cell count; } \\
\text { Hs-CRP, hypersensitive C-reactive protein. }\end{array}$} \\
\hline \multicolumn{10}{|c|}{ * $p$ value for difference between CKD and non-CKD participants } \\
\hline \multicolumn{10}{|c|}{ \& Interaction of sex by CKD status } \\
\hline
\end{tabular}

The visceral adiposity has a closely relationship with metabolic disorders. Thus, Table 4 adjusted for VAl instead of BMI as a represent of visceral adiposity. Women with CKD had significantly higher levels of TC, LDL-C, non-HDL-C and Apo B than their men counterparts with CKD. The differences of WBC between women with CKD and non-CKD ones was much more significantly than men. Also, women with CKD have higher level of Lp(a) and LAP compared with men with CKD. Besides, the magnitude of differences in $\mathrm{HbA1c}$, ApoB/Apo a1, FPG, Hs-CRP and WBC between different genders with CKD were less marked. The Hb of women with CKD was lower compared with men with CKD. The interactions of sex $\times$ CKD mentioned in Table 2 remained significant still. After adjusting the HOMA-IR (Table 5), the difference of these CVD factors between women with CKD and men with CKD remained essentially unchanged.

\section{Discussion}

This was a study of the differences between men and women in the comparison of traditional and "non-traditional" CVD markers in CKD patients. Compared with men, women were more likely to have an atherogenic lipid pattern and visceral obese in patients with CKD. Besides, in comparison with the non-CKD counterparts, women with CKD showed greater differences in the value of WBC than men with CKD. The sex differences effect on CVD risk factors of CKD patients and non-CKD ones can't be eliminated considering with BMI, VAI or HOMA-IR or both.

The level of traditional lipid markers, such as TC, were found to be positively associated with an increased risk of CVD, coronary heart disease (CHD) and cardiac death (CHD plus heart failure) (24). In this research, the value of TC in women with CKD was higher than men. Previous research showed that higher level of LDL-C, non-HDL-C and Apo B indicated a higher incidence of future cardiovascular events (25). In this study, the level of LDL-C, non-HDL-C and Apo B of women with CKD were higher than men with CKD as well, which indicate a worse situation of risk factors of CVD of women with CKD. Thus, the traditional lipid markers of women may need more attention. 
In this study, we observed that Lp (a) of women with CKD was significantly higher than that of men with CKD. Lp (a) is one of the cardiovascular factors that have been discovered and showed significantly positively correlated with CVD in previous studies (7). Besides, apolipoprotein(a) bound to apolipoprotein B of an LDL-like particle covalently composed Lp(a) (26) and it mediates atherogenicity by using its LDL moiety. Moreover, $L p(a)$ can induce the proinflammatory responses as one of its normal function(27). In previous studies, androgens had a greater affection of decreasing the level of $L p(a)$ compared with estrogens. (28).Besides, most of the women with CKD patients are old, and mostly are in menopause (14). Thus, there is a possibility of women with CKD have a worse situation of $L p(a)$ compared with men with CKD and we need to promote the realization of $L p$ (a) in women with CKD.

WBC is also one of the newly discovered cardiovascular risk factors, which participates various stages of cardiovascular disease progression and complication(29). Oxidative stress and inflammation were the important mechanisms for WBC to be considered as a risk factor for CVD in patients with CKD(30). In our study, the value of WBC increased in both women and men with CKD. Moreover, the difference between CKD and non-CKD women was significantly higher than men. It indicated that women may get more serious processes of inflammation from non-CKD to CKD compared with men.

VAl and LAP are important markers of visceral obesity (31) and we found that the level of VAI and LAP in women with CKD were higher than men with CKD in this study. Lots of studies showed that VAI and LAP are associated with a high risk of CVD (32). The INTERHEART study had showed that VAl contributes a lot to CVD risk, evaluating the impact of obesity on CVD(33). Also, woman was found as a risk factor of abdominal obesity in the previous study(34). The specific sex-based mechanisms are still unclear(35). Simultaneously, the LAP is considered as an better index compared with BMI to recognize CVD risk as an index which describes lipid over-accumulation based on WC and fasting triglycerides (36). In this study, compared with BMI, VAI and LAP were more sensitive to reflect the change of lipid pattern in CKD patients. Thus, paying more attention to these two factors instead of BMI only in CKD patients may help doctors to get awareness of CVD risk.

The reasons of the severe condition of the risk factors of CVD of women with CKD may be the following points. Frist, previous studies have shown that autoimmune diseases, pregnancy, dialysis and transplantation lead specific challenges to women with $\operatorname{CKD}(37,38)$. Second, it is not equal among men and women to access the medical care of CKD in lots of places around the world (39). Third, the lost protection of estrogen in women is also a dangerous factor for the risk of CVD in women with CKD(40). Finally, the progress of the times has made women no longer constrained by family life, but also faces more pressure from work and society, especially in China(41). This stress may increase the inflammation of women. It is important to keep the health of families, communities and populations to advocate for improving the access to medical for women (42). Therefore, more attention needed to pay to the risk factors of CVD of women with CKD to aware of the unsatisfactory condition of women with CKD.

Our study had some limitations at the same time. Firstly, it was difficult to explain whether the CKD in women will experience worse changes in visceral adiposity, some lipid profile and TyG during the process of CKD because of the cross-sectional study design. Second, since we only have the only one blood sample, the definition of CKD may not exactly as it showed. Finally, the causality between the greater relative CVD risk and these great change in the CVD risk factors among women with CKD was hard to find. However, there were also some strengths of our study, such as these data used in this study were population-based. Also, covariates of interest in this research were assessed in detail. Some non-traditional risk factors such as inflammation markers and visceral adiposity were included and they were calculated as well.

\section{Conclusions}

In summary, our study documented that CKD women had a tendency of having worse CVD risk factors than men. Increased CVD risk factors were observed in this study in both genders with CKD. CKD women had higher value of CVD risk factors than men. Moreover, the greater changes in some CVD risk factors of women with CKD compared with man were independent of important factors, such as $\mathrm{BMI}$, age, visceral adiposity or HOMA-IR. These important findings may help to suggest the increased relative CVD risk in CKD women compared with men.

\section{Abbreviations}




\begin{tabular}{|ll|}
\hline Abbreviations & \\
\hline CVD & Cardiovascular disease \\
\hline CKD & Chronic kidney disease \\
\hline Lp (a) & Lipoprotein (a) \\
\hline WBC & White blood cell \\
\hline VAI & Visceral adiposity index \\
\hline LAP & Lipid accumulation product \\
\hline CHNS & China Health and Nutrition Survey \\
\hline BMI & Body mass index \\
\hline TG & Triglycerides \\
\hline HDL-C & High density lipoprotein cholesterol \\
\hline WC & Waist circumference \\
\hline BP & Blood pressure \\
\hline WHO & World Health Organization \\
\hline FPG & Fasting plasm glucose \\
\hline HbA1c & Hemoglobin A1c \\
\hline TG & Triglycerides \\
\hline LDL-C & Low density lipoprotein cholesterol \\
\hline ALT & alanine aminotransferase \\
\hline UA & Hric acid \\
\hline hs-CRP & Hypersensitive C-reactive protein \\
\hline eGFR & \\
\hline HOMA-IR & Homeostasis model assessment of insulin resistance \\
\hline TyG & \\
\hline
\end{tabular}

\section{Declarations}

\section{Ethics approval and consent to participate:}

the institutional review committees of the University of North Carolina at Chapel Hill, the National Institute of Nutrition and Food Safety, Chinese Center for Disease Control and Prevention, and the China-Japan Friendship Hospital, Ministry of Health Each approved this survey and participant provided a written informed consent

\section{Consent for publication:}

Not applicable

\section{Availability of data and materials:}

The datasets generated and/or analysed during the current study are available in the China Health and Nutrition Survey repository, https://www.cpc.unc.edu/projects/china 


\section{Competing interests:}

The authors declare that they have no competing interests

\section{Funding:}

This research was supported by the International (regional) cooperation and exchange projects (NSFC-DFG, Grant No. 81761138041); National Natural Science Foundation of China (Grants 81570667, 81470948, 81670633); Major Research Plan of the National Natural Science Foundation of China (Grant No. 91742204); The National Key R\&D Program of China (Gran2018YFC1314003-1ه2015BAl12B07), and National key research and development program (Grants 2016YFC0906103).

\section{Author contributions:}

ZFW and NHZ had full access to all of the data in the study and takes responsibility for the accuracy of the data analysis. SWG, GX and ZFW developed study concept and design. WZF, NHZ, RL, CYC, CD, and TTL performed the statistical analyses, and drafted the manuscript. GX and SWG obtained the funding and supervised the study.

\section{Acknowledgments:}

We acknowledge and thank the China Health and Nutrition Survey, supported by the NIH (R01-HD30880, DK056350 and R01HD38700), and the National Institute of Nutrition and Food Safety, China Center for Disease Control and Prevention, Carolina Population Center, the University of North Carolina at Chapel Hill and the Fogarty International Center for providing the data used here. We also thank the China-Japan Friendship Hospital and Ministry of Health for support for CHNS 2009 survey.

\section{References}

1. Ng JKC, Li PKT. Chronic kidney disease epidemic: How do we deal with it? Nephrology (Carlton). 2018;23Suppl 4:116-20.

2. Zhang L, Wang F, Wang L, Wang W, Liu B, Liu J, et al. Prevalence of chronic kidney disease in China: a cross-sectional survey. Lancet. 2012;379(9818):815-22.

3. Segall L, Nistor I, Covic A. Heart failure in patients with chronic kidney disease: a systematic integrative review. Biomed Res Int. 2014;2014:937398.

4. Vallianou NG, Mitesh S, Gkogkou A, Geladari E. Chronic Kidney Disease and Cardiovascular Disease: Is there Any Relationship? Curr Cardiol Rev. 2019;15(1):55-63.

5. Liu M, Li XC, Lu L, Cao Y, Sun RR, Chen S, et al. Cardiovascular disease and its relationship with chronic kidney disease. Eur Rev Med Pharmacol Sci. 2014;18(19):2918-26.

6. Ronco C, Bellasi A, Di Lullo L. Cardiorenal Syndrome: An Overview. Advances in chronic kidney disease. 2018;25(5):382-90.

7. Kotseva K, Wood D, De Backer G, De Bacquer D, Pyorala K, Keil U. EUROASPIRE III: a survey on the lifestyle, risk factors and use of cardioprotective drug therapies in coronary patients from 22 European countries. European journal of cardiovascular prevention rehabilitation: official journal of the European Society of Cardiology Working Groups on Epidemiology Prevention Cardiac Rehabilitation Exercise Physiology. 2009;16(2):121-37.

8. Maranhao RC, Carvalho PO, Strunz CC, Pileggi F. Lipoprotein (a): structure, pathophysiology and clinical implications. Arquivos brasileiros de cardiologia. 2014;103(1):76-84.

9. Sun J, Axelsson J, Machowska A, Heimburger O, Barany P, Lindholm B, et al. Biomarkers of Cardiovascular Disease and Mortality Risk in Patients with Advanced CKD. Clinical journal of the American Society of Nephrology: CJASN. 2016;11(7):1163-72.

10. Appelman Y, van Rijn BB, Ten Haaf ME, Boersma E, Peters SA. Sex differences in cardiovascular risk factors and disease prevention. Atherosclerosis. 2015;241(1):211-8. 
11. Fan H, Li X, Zheng L, Chen X, Lan Q, Wu H, et al. Abdominal obesity is strongly associated with Cardiovascular Disease and its Risk Factors in Elderly and very Elderly Community-dwelling Chinese. Scientific reports. 2016;6:21521.

12. Zhou C, Peng H, Yuan J, Lin X, Zha Y, Chen H. Visceral, general, abdominal adiposity and atherogenic index of plasma in relatively lean hemodialysis patients. BMC Nephrol. 2018;19(1):206.

13. Kaplan A, Abidi E, Ghali R, Booz GW, Kobeissy F, Zouein FA. Functional, Cellular, and Molecular Remodeling of the Heart under Influence of Oxidative Cigarette Tobacco Smoke. Oxidative medicine and cellular longevity. 2017;2017:3759186.

14. Link JC, Reue K. Genetic Basis for Sex Differences in Obesity and Lipid Metabolism. Ann Rev Nutr. 2017;37:225-45.

15. Du XJ, Fang L, Kiriazis H. Sex dimorphism in cardiac pathophysiology: experimental findings, hormonal mechanisms, and molecular mechanisms. Pharmacol Ther. 2006;111(2):434-75.

16. Carrero JJ, Hecking M, Chesnaye NC, Jager KJ. Sex and gender disparities in the epidemiology and outcomes of chronic kidney disease. Nature reviews Nephrology. 2018;14(3):151-64.

17. Du T, Yuan G, Zhou X, Sun X. Sex differences in the effect of HbA1c-defined diabetes on a wide range of cardiovascular disease risk factors. Ann Med. 2016;48(1-2):34-41.

18. Popkin BM, Du S, Zhai F, Zhang B. Cohort Profile: The China Health and Nutrition Survey-monitoring and understanding socioeconomic and health change in China, 1989-2011. Int J Epidemiol. 2010;39(6):1435-40.

19. Ma YC, Zuo L, Chen JH, Luo Q, Yu XQ, Li Y, et al. Modified glomerular filtration rate estimating equation for Chinese patients with chronic kidney disease. Journal of the American Society of Nephrology: JASN. 2006;17(10):2937-44.

20. Erratum: Kidney Disease: Improving Global Outcomes (KDIGO) CKD-MBD Update Work Group. KDIGO 2017 Clinical Practice Guideline Update for the Diagnosis, Evaluation, Prevention, and Treatment of Chronic Kidney Disease-Mineral and Bone Disorder (CKD-MBD). Kidney Int Suppl. 2017;7:1-59. Kidney international supplements. 2017;7(3):e1.

21. Ma A, Liu F, Wang C, Liang K, Yan F, Hou X, et al. Both insulin resistance and metabolic syndrome accelerate the progression of chronic kidney disease among Chinese adults: results from a 3-year follow-up study. Int Urol Nephrol. 2018;50(12):2239-44.

22. Guerrero-Romero F, Simental-Mendia LE, Gonzalez-Ortiz M, Martinez-Abundis E, Ramos-Zavala MG, Hernandez-Gonzalez SO, et al. The product of triglycerides and glucose, a simple measure of insulin sensitivity. Comparison with the euglycemichyperinsulinemic clamp. J Clin Endocrinol Metab. 2010;95(7):3347-51.

23. Amato MC, Giordano C, Galia M, Criscimanna A, Vitabile S, Midiri M, et al. Visceral Adiposity Index: a reliable indicator of visceral fat function associated with cardiometabolic risk. Diabetes Care. 2010;33(4):920-2.

24. Sugiyama D, Okamura T, Watanabe M, Higashiyama A, Okuda N, Nakamura Y, et al. Risk of hypercholesterolemia for cardiovascular disease and the population attributable fraction in a 24-year Japanese cohort study. J Atheroscler Thromb. 2015;22(1):95-107.

25. Ridker PM, Rifai N, Cook NR, Bradwin G, Buring JE. Non-HDL cholesterol, apolipoproteins A-I and B100, standard lipid measures, lipid ratios, and CRP as risk factors for cardiovascular disease in women. Jama. 2005;294(3):326-33.

26. Tsimikas S, Fazio S, Ferdinand KC, Ginsberg HN, Koschinsky ML, Marcovina SM, et al. NHLBI Working Group Recommendations to Reduce Lipoprotein(a)-Mediated Risk of Cardiovascular Disease and Aortic Stenosis. J Am Coll Cardiol. 2018;71(2):177-92.

27. van der Valk FM, Bekkering S, Kroon J, Yeang C, Van den Bossche J, van Buul JD, et al. Oxidized Phospholipids on Lipoprotein(a) Elicit Arterial Wall Inflammation and an Inflammatory Monocyte Response in Humans. Circulation. 2016;134(8):611-24.

28. Gaeta G, Lanero S, Barra S, Silvestri N, Cuomo V, Materazzi C, et al. Sex hormones and lipoprotein(a) concentration. Expert Opin Investig Drugs. 2011;20(2):221-38.

29. Swirski FK, Nahrendorf M. Leukocyte behavior in atherosclerosis, myocardial infarction, and heart failure. Science. 2013;339(6116):161-6.

30. Bulbul MC, Dagel T, Afsar B, Ulusu NN, Kuwabara M, Covic A, et al. Disorders of Lipid Metabolism in Chronic Kidney Disease. Blood Purif. 2018;46(2):144-52.

31. Li R, Li Q, Cui M, Ying Z, Li L, Zhong T, et al. Visceral adiposity index, lipid accumulation product and intracranial atherosclerotic stenosis in middle-aged and elderly Chinese. Scientific reports. 2017;7(1):7951-.

32. Kouli GM, Panagiotakos DB, Kyrou I, Georgousopoulou EN, Chrysohoou C, Tsigos C, et al. Visceral adiposity index and 10-year cardiovascular disease incidence: The ATTICA study. Nutrition, metabolism, and cardiovascular diseases. NMCD.

Page $17 / 18$ 
2017;27(10):881-9.

33. Yusuf S, Hawken S, Ounpuu S, Dans T, Avezum A, Lanas F, et al. Effect of potentially modifiable risk factors associated with myocardial infarction in 52 countries (the INTERHEART study): case-control study. Lancet. 2004;364(9438):937-52.

34. Chew WF, Leong PP, Yap SF, Yasmin AM, Choo KB, Low GKK, et al. Risk factors associated with abdominal obesity in suburban adolescents from a Malaysian district. Singapore Med J. 2018;59(1):104-11.

35. Liu R-Q, Qian Z, Wang S-Q, Vaughn MG, Geiger SD, Xian H, et al. Sex-Specific Difference in the Association Between Poor Sleep Quality and Abdominal Obesity in Rural Chinese: A Large Population-Based Study. J Clin Sleep Med. 2017;13(4):565-74.

36. Kyrou I, Panagiotakos DB, Kouli GM, Georgousopoulou E, Chrysohoou C, Tsigos C, et al. Lipid accumulation product in relation to 10-year cardiovascular disease incidence in Caucasian adults: The ATTICA study. Atherosclerosis. 2018;279:10-6.

37. Piccoli GB, Cabiddu G, Attini R, Vigotti FN, Maxia S, Lepori N, et al. Risk of Adverse Pregnancy Outcomes in Women with CKD. Journal of the American Society of Nephrology: JASN. 2015;26(8):2011-22.

38. Ortona E, Pierdominici M, Maselli A, Veroni C, Aloisi F, Shoenfeld Y. Sex-based differences in autoimmune diseases. Ann Ist Super Sanita. 2016;52(2):205-12.

39. Piccoli GB, Alrukhaimi M, Liu Z-H, Zakharova E, Levin A. World Kidney Day Steering C. Women and kidney disease: Reflections on world kidney day 2018. J Ren Care. 2018;44(1):3-11.

40. Xing D, Nozell S, Chen Y-F, Hage F, Oparil S. Estrogen and mechanisms of vascular protection. Arterioscler Thromb Vasc Biol. 2009;29(3):289-95.

41. Blom M, Janszky I, Balog P, Orth-Gomer K, Wamala SP. Social relations in women with coronary heart disease: the effects of work and marital stress. J Cardiovasc Risk. 2003;10(3):201-6.

42. Bina R, Glasser S. Factors associated with attitudes toward seeking mental health treatment postpartum. Women Health. 2019;59(1):1-12. 